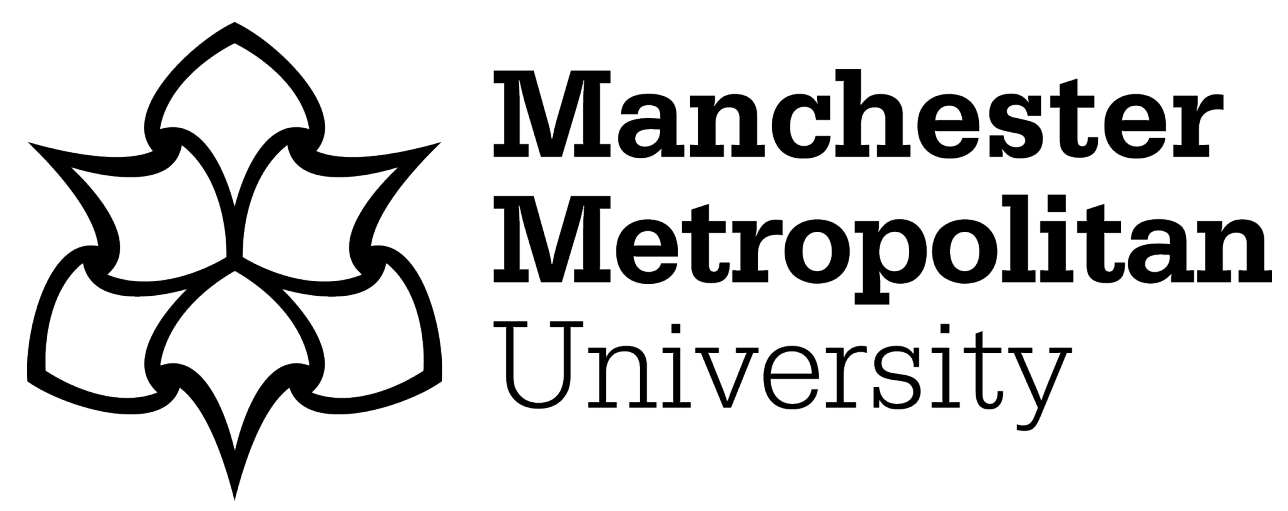

Zwolinsky, S, McKenna, J, Pringle, A, Widdop, P ORCID logoORCID: https://orcid.org/0000-0003-0334-7053, Griffiths, C, Mellis, M, Rutherford, Z and Collins, P (2016) Physical activity and sedentary behavior clustering: Segmentation to optimize active lifestyles. Journal of Physical Activity and Health, 13 (9). pp. 921-928. ISSN 1543-3080

Downloaded from: https://e-space.mmu.ac.uk/624974/

Version: Accepted Version

Publisher: Human Kinetics

DOI: https://doi.org/10.1123/jpah.2015-0307

Please cite the published version 
See discussions, stats, and author profiles for this publication at: https://www.researchgate.net/publication/302982746

\section{Physical Activity and Sedentary Behavior Clustering: Segmentation to Optimize Active Lifestyles}

Article in Journal of physical activity \& health · May 2016

Dol: 10.1123/jpah.2015-0307

CITATIONS

7

8 authors, including:

Stephen Zwolinsky

West Yorkshire \& Harrogate Cancer Alliance

50 PUBLICATIONS 429 CITATIONS

SEE PROFILE

Andy Pringle

University of Derby

88 PUBLICATIONS 468 CITATIONS

SEE PROFILE
READS

243

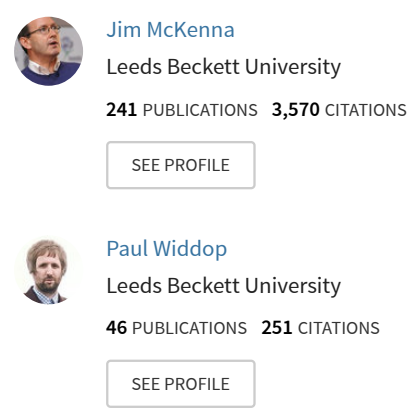

Some of the authors of this publication are also working on these related projects:

Development of a modified version of Primary Rugby League (with the Rugby Football League) View project

Physical Activity and Women: Reaching the 'Hard to Reach' (PhD) View project 
"Physical Activity and Sedentary Behaviour Clustering: Segmentation to Optimise Active Lifestyles" by Zwolinsky S et al. Journal of Physical Activity \& Health

(C) 2016 Human Kinetics, Inc.

Note: This article will be published in a forthcoming issue of the Journal of Physical Activity \& Health. This article appears here in its accepted, peer-reviewed form, as it was provided by the submitting author. It has not been copy edited, proofed, or formatted by the publisher.

Section: Original Research

Article Title: Physical Activity and Sedentary Behaviour Clustering: Segmentation to Optimise Active Lifestyles

Authors: Stephen Zwolinsky, James McKenna, Andy Pringle, Paul Widdop, Claire Griffiths, Michelle Mellis, Zoe Rutherford, and Peter Collins

Affiliations: Centre for Active Lifestyles, Leeds Beckett University, Leeds, UK.

Running Head: Physical activity and sedentary behaviour clustering

Journal: Journal of Physical Activity \& Health

Acceptance Date: March 12, 2016

(C)2016 Human Kinetics, Inc.

DOI: $\underline{\text { http://dx.doi.org/10.1123/jpah.2015-0307 }}$ 
"Physical Activity and Sedentary Behaviour Clustering: Segmentation to Optimise Active Lifestyles" by Zwolinsky S et al. Journal of Physical Activity \& Health

(C) 2016 Human Kinetics, Inc.

\section{ABSTRACT}

Background: Increasingly the health impacts of physical inactivity are being distinguished from those of sedentary behaviour. Nevertheless, deleterious health prognoses occur when these behaviours combine, making it a Public Health priority to establish the numbers and salient identifying factors of people who live with this injurious combination. Method: Using an observational between-subjects design, a non-probability sample of 22,836 participants provided data on total daily activity. A 2-step hierarchical cluster analysis identified the optimal number of clusters and the subset of distinguishing variables. Univariate analyses assessed significant cluster differences. Results: High levels of sitting clustered with low physical activity. The 'Ambulatory \& Active' cluster $(\mathrm{n}=6,254)$ sat for 2.5 to $5 \mathrm{~h} \mathrm{~d}^{-1}$ and were highly active. They were significantly younger, included a greater proportion of males and reported low Indices of Multiple Deprivation compared to other clusters. Conversely, the 'Sedentary \& Low Active' cluster $(n=6,286)$ achieved $\leq 60$ MET. min. wk $^{-1}$ of physical activity and sat for $\geq 8 \mathrm{~h} \mathrm{~d}^{-1}$. They were the oldest cluster, housed the largest proportion of females and reported moderate Indices of Multiple Deprivation. Conclusions: Public Health systems may benefit from developing policy and interventions that do more to limit sedentary behaviour and encourage light intensity activity in its place.

Keywords: physical activity assessment, sedentary behaviour, public health

Abstract Work Count: 200

Manuscript Word Count: 4035 
"Physical Activity and Sedentary Behaviour Clustering: Segmentation to Optimise Active Lifestyles" by Zwolinsky S et al. Journal of Physical Activity \& Health

(C) 2016 Human Kinetics, Inc.

\section{INTRODUCTION:}

Empirical evidence underpinning the positive associations found between moderate to vigorous physical activity (MVPA) and health are longstanding. ${ }^{1}$ What is more, growing epidemiological and physiological research has highlighted adverse health implications for prolonged sedentary time, regardless of activity status. ${ }^{2-5}$ The UK physical activity guidelines now recommend that individuals should minimise daily sedentary time. ${ }^{6}$ However, estimates from objective monitoring indicate that adults spend around 7-9 hours of their working day sedentary. ${ }^{6-8}$ Consequently, strategies that identify at-risk groups, reduce sedentary behaviour and increase MVPA have become a Public Health priority. ${ }^{9-12}$

Developing our understanding of sedentary behaviour is crucial for better Public Health. Initially sedentary behaviour was simply viewed as being at the lower end of the physical activity continuum, whereas, it actually refers to a group of waking behaviours primarily characterised by sitting and low energy expenditure. ${ }^{3}$ Waking behaviours including sitting, reclining or standing - at the required intensities - can be sedentary. ${ }^{13,14}$ However, there are physiological differences between these activities. For example, being sedentary while standing does not have the same negative metabolic consequences as sitting. ${ }^{15}$ Sit to stand interventions can elicit significant increases in energy expenditure among obese/overweight office workers providing an interesting avenue for intervention. ${ }^{16}$ Furthermore, although sedentary behaviour and sitting clearly overlap, they are not synonymous. ${ }^{9}$ This illustrates the complex and nuanced nature of sedentary behaviour research.

Regardless of MVPA levels, extended doses of sedentary behaviour can increase an individual's risk of developing the metabolic syndrome, ${ }^{17}$ type 2 diabetes ${ }^{18-20}$ and obesity. ${ }^{21,22}$ This seems to be in some way determined by variations in lipoprotein lipase (LPL) activity - an 
"Physical Activity and Sedentary Behaviour Clustering: Segmentation to Optimise Active Lifestyles" by Zwolinsky S et al. Journal of Physical Activity \& Health

(C) 2016 Human Kinetics, Inc.

enzyme that enables the uptake of free fatty acids into skeletal muscle and adipose tissue. ${ }^{15}$ Worryingly, data suggests that both acute and prolonged sedentary behaviour may lower LPL activity. ${ }^{5}$ Further, higher levels of sedentary behaviour increases the odds of presenting multiple lifestyle risk factors, ${ }^{23}$ premature cardiovascular disease and all-cause mortality. ${ }^{18-}$ ${ }^{20,24-26}$ Another recognised effect of sedentary behaviour is a lowering in bone mineral density, which increases the risk of osteoporosis in older age. ${ }^{27,28}$

Numerous studies have shown only weak correlations between MVPA and sedentary behaviour. 22,26,29 Yet in Public Health terms, a 'perfect storm' occurs with a confluence of low activity and high sedentary behaviour. ${ }^{9}$ However, it is unwise to assume that interventions or policy influence both behaviours equally. ${ }^{30}$ For some groups, strategies that focus on improvements in these behaviours simultaneously are effective. ${ }^{31}$ Nevertheless, research surrounding effective intervention and policy to achieve this end is not well developed. ${ }^{3,32}$ The latest evidence indicates that interventions aiming to increase MVPA, or combine increases in MVPA with reductions in sedentary behaviour are not effective for reducing sedentary time. ${ }^{32}$

Better Public Health may hinge on our understanding of how these behaviours displace one another. For example, sedentary time is thought to displace total daily activity time, which combines MVPA with light intensity or non-exercise activity. ${ }^{2,33,34}$ At a population level, data points towards sedentary behaviour primarily displacing light intensity activity, ${ }^{22}$ which also has considerable benefits for cardio-metabolic health. ${ }^{35-37}$ At another level, emerging evidence has suggested that, in young people at least, these behaviours do not directly displace one another. ${ }^{38}$ Nevertheless, determining which physical activities and intensities sedentary behaviour displaces, if any, is paramount. 
"Physical Activity and Sedentary Behaviour Clustering: Segmentation to Optimise Active Lifestyles" by Zwolinsky S et al. Journal of Physical Activity \& Health

(C) 2016 Human Kinetics, Inc.

Sedentary behaviour is an irreducible component of modern lifestyles, irrespective of activity status. Yet, a paucity of evidence exists that explores how these behaviours coalesce. The aim of this paper is to strengthen our knowledge of the relationship between sedentary behaviour and physical activity, understand more about how these behaviours cluster and examine how individuals can be classified through shared behaviours and characteristics. Findings from this paper may help to refine the methods used to locate individuals who uphold the worst behavioural combinations and aid the development of theoretically underpinned interventions that help people to substitute unhealthy behaviours for more favourable ones. ${ }^{10,39}$

\section{METHODS:}

\section{Study Population}

The 'Leeds Let's Get Active' (LLGA) intervention was developed by Leeds City Council as part of Sport England's 'Get Healthy, Get into Sport' funding stream. LLGA is a community physical activity intervention that encourages inactive Leeds residents to 'do more activity'. Participants engaging the scheme have free access to 17 Leeds City Council leisure centre swimming pools and gyms on specified days and times. Each week around 150 hour long timetabled sessions - predominantly off-peak - are available across the participating sites. Recruitment was open to all adults in Leeds (Yorkshire, UK). Prior to engaging LLGA sessions, participants were given a standard leisure centre induction.

This study employed an observational between-subjects design using a nonprobability sample. Participants enrolling in the scheme completed baseline measures either on-line or using paper based methods. The total data set consisted of 31,737 respondents. Participants were excluded from the analysis if they were aged $<16$ years old $(n=3,333)$ or 
"Physical Activity and Sedentary Behaviour Clustering: Segmentation to Optimise Active Lifestyles" by Zwolinsky S et al. Journal of Physical Activity \& Health

(C) 2016 Human Kinetics, Inc.

their contributions failed to correspond to the data cleaning guidelines of the baseline measures $(n=5,568)$. The resulting data set contained 22,836 participants. Ethical approval was obtained through Leeds Beckett University research ethics committee and all participants provided informed consent. Data collection took place between September 2013 and December 2014.

\section{Measures}

Self-reported data were captured through a short self-reported survey. Notwithstanding their recognised limitations, self-report measures are still the most pragmatic and frequently used solution for large-scale population research and surveillance. ${ }^{21}$ Further, much of the research examining physical activity and sitting time behaviour is underpinned by self-report as the principal data collection method. ${ }^{40,41}$

Indices of Multiple Deprivation (IMD) scores were determined using residential postcode data. IMD scores provide a relative, continuous measure of deprivation at lower super output area (LSOA) level. Areas are ranked on seven different dimensions of deprivation to determine an overall composite measure of multiple deprivation. Across England, IMD is calculated for every LSOA, or neighbourhood. Each LSOA is ranked according to its level of deprivation relative to that of other areas. ${ }^{42}$ Whilst LSOA's are not categorised as deprived or not by an arbitrary number, IMD scores were split into deciles. Participants residing within the bottom $20 \%$ were classified as 'most deprived', and the remaining $80 \%$ were classed as 'least deprived'. ${ }^{43}$

Physical activity data were captured using the short version International Physical Activity Questionnaire (sIPAQ). ${ }^{44}$ sIPAQ is a short recall questionnaire providing a quick assessment of the total volume of physical activity classified by dimension of intensity or 
"Physical Activity and Sedentary Behaviour Clustering: Segmentation to Optimise Active Lifestyles" by Zwolinsky S et al. Journal of Physical Activity \& Health

(C) 2016 Human Kinetics, Inc.

domain (type or mode). sIPAQ demonstrates good reliability and moderate criterion validity for use with community residing adults. ${ }^{44,45}$ MET.min.wk ${ }^{-1}$ expenditure was calculated by summing the relevant activity dimension's and domains. Participants were classified in to one of three physical activity categories; (i) low ( $\leq 599$ MET.min.wk ${ }^{-1}$ ), (ii) moderate $(600-2,999$ MET.min.wk-1 or (iii) high $\left(3,000+\right.$ MET.min. $\left.w^{-1}\right) \cdot{ }^{46}$

The measure of sedentary behaviour used in this study was weekday sitting time assessed through the sIPAQ. While this is a validated measure, ${ }^{44}$ recent evidence has shown that sIPAQ or 7 day recall can grossly underestimate sitting time. ${ }^{11,47}$ Nevertheless, the line of questioning adopted here underpins many large scale sitting time surveys. ${ }^{21}$ Resulting scores were divided into quintiles as thresholds for increased sitting risk have not yet been established.

Physical activity data were cleaned according to the data processing rules of the sIPAQ scoring protocol. ${ }^{46}$ There are no established criteria for cleaning or truncating the sitting item question for analysis. However, in this study, to remove missing and spurious measures of sitting time, individuals leaving the sitting item blank, reporting zero or $>16$ hours of sitting time per day were excluded from the analysis.

\section{Cluster analysis}

Clustering is a form of multivariate analysis that can assess the co-occurrence of risk behaviours. ${ }^{48}$ Two-step cluster analyses are designed to handle large data sets that contain both categorical and continuous data. Therefore, this technique was employed to group respondents in this study. No assumptions were made about the number of clusters or cluster membership prior to analysis. The first step assigned cases to pre-clusters and the second 
"Physical Activity and Sedentary Behaviour Clustering: Segmentation to Optimise Active Lifestyles" by Zwolinsky S et al. Journal of Physical Activity \& Health

(C) 2016 Human Kinetics, Inc.

step clustered the pre-clusters using a hierarchical procedure. The optimal number of clusters was determined by an algorithm based on the Schwarz Bayesian Criterion. ${ }^{49}$

Multiple iterations of the cluster analysis were undertaken to validate the optimum number of clusters and the subset of variables to be included. The following variables derived from the self-report measures - were included in the final clustering solution (i) sitting quintile, (ii) activity category, (iii) total vigorous MET's, (iv) total moderate METs and, (v) total walking METs. All variables were standardised and the log-likelihood criterion was employed. To confirm the findings in the final solution, a split-half cross validation was used.

\section{Statistical Methods}

To establish the distinctiveness of each cluster, univariate analyses, including Pearson's Chi-square tests assessed for significant associations between categorical variables (gender, age group and IMD decile) and cluster membership. To determine effect size, Cramer's V coefficient and omega squared $(\omega)$ measured of the strength of the association. One-way ANOVA's compared mean scores by cluster membership across continuous variables (age). For all inferential tests, a $p$ value of $<.05$ was taken to be statistically significant. Data analyses were undertaken using IBM SPSS Statistics v21.

\section{RESULTS:}

\section{Demographic Characteristics (Table 1)}

Variations in sample size for demographic characteristics are a result of incomplete data capture. Data on gender were available for 14,405 (63.1\%) women and 8,408 (36.9\%) men. The mean age of the participants was 38.8 years $(\sigma=14.9)$ and $70.6 \%$ were aged $\leq 45$ years. Indices of Multiple Deprivation (IMD) scores ranged from $1.25-75.71$. Using IMD 
"Physical Activity and Sedentary Behaviour Clustering: Segmentation to Optimise Active Lifestyles" by Zwolinsky S et al. Journal of Physical Activity \& Health

(C) 2016 Human Kinetics, Inc.

scores and defining deprivation as living in the top 20\% deprived areas, 4,313 adults were categorised as 'most deprived'.

\section{Physical Activity and Sitting Time Behaviour}

In total, a median value of $1,480 \mathrm{MET} \cdot \mathrm{min} \cdot \mathrm{wk}^{-1}$ of physical activity were reported by participants; the mean value was 2,504 MET.min. $\mathrm{wk}^{-1}(\sigma=2,904.9)$. Data from participants providing sitting time and activity data shows that $27.9 \%$ were classified as low active, $44.5 \%$ were classified as moderately active and $27.5 \%$ were classified as high active. There was a significant association between activity category and gender $\left(\chi^{2}[2]=352.465, p<.001\right)$, and activity category and deprivation $\left(\chi^{2}[2]=86.864, p<.001\right)$. Males (Cramer's $\left.V=0.124\right)$ and the 'most deprived' group (Cramer's $V=0.062$ ) reported the most preferable activity categories. Even though there was a significant association, the effect sizes were moderate and low respectively. There was a significant effect for age on activity category $(F[2,22833]=87.075$, $p<.001, \omega=.09)$. Participants in the high activity category were significantly younger than all other age groups $(p<.001)$.

A median value of 300 min. $\mathrm{d}^{-1}$ of sitting were reported by all participants; the mean value was 317.5 min. $^{-1}(\sigma=173.65)$, equal to approximately 5 hours per day. Quintiles of daily sitting time ranged from $0-150$ minutes in the lowest quintile, to $\geq 481$ minutes in the highest quintile. There were significant associations between sitting quintiles and gender $\left(\chi^{2}\right.$ $[4]=24.210, p<.001)$, and sitting quintiles and deprivation $\left(\chi^{2}[4]=64.441, p<.001\right)$. Females (Cramer's $V=0.033$ ) and the 'most deprived' group (Cramer's $V=0.054$ ) reported the lowest sitting quintiles and therefore the lowest sitting time. There was a significant effect for age on sitting quintile $(F[4,22563]=69.095, p<.001, \omega=.11)$. Participants in the lowest sitting 
"Physical Activity and Sedentary Behaviour Clustering: Segmentation to Optimise Active Lifestyles" by Zwolinsky S et al. Journal of Physical Activity \& Health

(C) 2016 Human Kinetics, Inc.

quintile were significantly younger than all other age groups $(p<.001)$. Although all associations were significant, effect sizes were low.

Figure 1 shows the prevalence of sitting quintile by physical activity category determined by sIPAQ for all participants. Data indicates that low active participants were most likely to present higher sitting quintiles, with $48.7 \%$ of low active participants categorised in sitting quintiles four or five. Further, high active participants were more likely to present lower sitting quintiles, with $54.2 \%$ of high active participants categorised in quintiles one or two.

\section{Cluster Analysis}

A three cluster solution was identified based on maximising the similarity within the clusters and variability between clusters. This was validated in a split-half sample. The silhouette measure, which can be used to interpret and validate the solution, was 0.5 . This represents good cohesion and separation between the clusters. There were $27.7 \%(n=6,254)$ participants assigned to cluster $1,44.4 \%(n=10,028)$ participants assigned to cluster 2 and $27.9 \%(6,286)$ to cluster 3 . The ratio of sizes between the clusters was 1.60 .

Descriptive characteristics of the cluster profiles can be seen in table 2. Cluster 1 (Ambulatory \& Active) exhibited the lowest sitting quintile, sitting for around 151-240 min. $\mathrm{d}^{-}$ ${ }^{1}$. They were also classified as high active by sIPAQ. This differs to cluster 2 (Moderation) who were classified as moderately active by SIPAQ and in sitting quintile 3, sitting for 241-630 min. $d^{-1}$. In contrast, cluster 3 (Sedentary \& Low Active) presented the highest sitting quintile, sitting for $\geq 481$ min. $^{-1}$ and was classified as low active by sIPAQ. Cluster 1 were undertaking the most vigorous activity each week, further it was the only cluster where vigorous activity made up the greatest contribution to total weekly activity, $40.9 \%$. For cluster 2 , vigorous 
"Physical Activity and Sedentary Behaviour Clustering: Segmentation to Optimise Active Lifestyles" by Zwolinsky S et al. Journal of Physical Activity \& Health

(C) 2016 Human Kinetics, Inc.

activity accounted for $28.8 \%$ of total activity, and for cluster 3 it was $10.7 \%$. In cluster 3 , walking accounted for $75 \%$ of total weekly activity. For cluster 2 , walking was also the most prominent contributor to total activity, 53.3\%. However in cluster 1 walking only accounted for $35.8 \%$ of total activity (Table 2 ).

Table 3 shows the differences in demographic profiles between the three clusters. A moderate association was found between gender and cluster membership $\left(\chi^{2}[2]=323.991\right.$, $p<.001$, Cramers $V=.120)$. Cluster $3(69.2 \%)$ and cluster $2(65.3 \%)$ had the highest percentage of females, whereas cluster 1 (54.4\%) had the lowest proportion of females. There was a significant effect for cluster membership on age $(F[2,13503]=79.042, p<.001, \omega=.08)$. The Games-Howell post hoc test revealed that cluster 1 (37.1 years, $\sigma 15.56$ ) was significantly younger than cluster 2 (39.0 years, $\sigma 14.89) p<.001$, and cluster $3(40.5$ years, $\sigma 14.36) p<.001$. There were $53.3 \%$ of participants in cluster 1 aged $\leq 35$ years compared to $47.9 \%$ in cluster 2 and $42.3 \%$ in cluster 3 . Further, cluster 2 was significantly younger than cluster $3(p<.001)$. There was a significant effect for cluster membership on IMD score $(F[2,13147]=72.362$, $p<.001, \omega=.08)$. The Games-Howell post hoc test revealed that cluster 1 had a significantly higher IMD score compared to cluster $2(p<.001)$, and cluster $3(p<.001)$. There were $22.7 \%$ of participants in cluster 1 classified as most deprived compared to $17.1 \%$ in cluster 2 , and $18.8 \%$ in cluster 3. Further, cluster 3 displayed a significantly higher IMD score compared to cluster $2(p<.001)$

\section{DISCUSSION:}

This study adopted an original approach to understanding how people can be classified according to similarities in MVPA and sedentary behaviour using a large sample of UK adults. The main finding of this study indicated that high levels of self-reported sedentary 
"Physical Activity and Sedentary Behaviour Clustering: Segmentation to Optimise Active Lifestyles" by Zwolinsky S et al. Journal of Physical Activity \& Health

(C) 2016 Human Kinetics, Inc.

behaviour, determined by sitting time, appeared to cluster with low levels of MVPA. Although these behaviours are independent of each other, this finding supports research that there is ample time among waking hours for sedentary behaviour and inactivity to co-occur. ${ }^{33}$ The cluster of 'Sedentary \& Low Active' participants was typically older, female and presented moderate levels of deprivation compared to other clusters. Importantly, the clusters identified by this research are distinct and amenable to targeted Public Health campaigns. Given the associated health implications, reducing sedentary behaviour, especially for inactive individuals, is not only a major Public Health challenge, but a best buy for scarce Public Health resources. ${ }^{6}$

In this study, participants reported a median value of $5 \mathrm{~h} \mathrm{~d}^{-1}$ sitting time, which is similar to self-reported daily sitting time from other population-based UK data. ${ }^{8}$ Further, our data confirmed previous findings that participants in the lowest sitting groups are typically younger than those individuals who sit for longer periods. ${ }^{11}$ Women sat for more time each day compared to men, a gendered effect not noted in previous research. ${ }^{11}$ It has been documented that higher levels of sitting time appear to have a damaging influence on insulin resistance and chronic low grade inflammation in women but not men. ${ }^{50}$ Therefore, reducing sedentary time in these women should be a priority. To our knowledge, the association between sitting time with IMD has not been reported elsewhere. An unexpected finding from this study was that lower sitting quintiles were reported by the most deprived participants. This finding may in part be due to the non-probability sampling framework and/or the structure of the LLGA programme which offers predominantly 'off-peak' activity sessions. The 'off-peak' nature of the intervention may have attracted participants who were either unemployed, shift-workers, in full-time education or retired. These individuals are more likely 
"Physical Activity and Sedentary Behaviour Clustering: Segmentation to Optimise Active Lifestyles" by Zwolinsky S et al. Journal of Physical Activity \& Health

(C) 2016 Human Kinetics, Inc.

to present lower IMD scores and accumulate lower, or no occupational sitting time, which may account for a large proportion of the weekday sitting time assessed by the sIPAQ.

The 'Sedentary \& Low Active' cluster presented the most problematic activity profile. Categorised as low active by SIPAQ, participants in this cluster were undertaking $<60$ MET.min.wk $\mathrm{k}^{-1}$ and sitting for more than $8 \mathrm{~h} \mathrm{~d}^{-1}$. Previous studies have shown that one day of uninterrupted sitting can cause a $39 \%$ reduction in insulin activity, even in healthy adults. ${ }^{51}$ When combined with low activity these characteristics produce a deleterious matrimony. ${ }^{52}$ Recent research has shown that compared to sitting, simply alternating between sitting and standing every 30 minutes can lead to significant increases in energy expenditure ${ }^{16}$ and cardio-metabolic health. ${ }^{22}$ These effects are thought to be, in part, elicited by engaging the large muscle groups in the lower body. ${ }^{15}$ Nevertheless, effective interventions for individuals and groups within this cluster are scarce. ${ }^{32}$ Only by improving the quality and consistency of theory-driven interventions, ${ }^{39}$ combined with improvements in measurement and understanding will we begin to control and improve behaviour.

Public Health practitioners are unlikely to optimise health by promoting MVPA to 'Sedentary \& Low Active' individuals, despite the well-established health benefits of being 'Ambulatory \& Active'. ${ }^{6}$ Accepting that stimuli for undertaking MVPA are not synonymous with stimuli for reducing sedentary behaviour will be critical for generating positive change. ${ }^{3,30}$ For individuals who are not receptive to organised or structured programmes of MVPA, reducing sedentary behaviour and increasing light activity may be a more viable approach or proximal goal for increasing movement and energy expenditure. This approach may help to break up prolonged bouts of sedentary time and elicit a range of unique healthrelated benefits. ${ }^{36}$ Even if action leads to small increases in physical activity there is potential 
"Physical Activity and Sedentary Behaviour Clustering: Segmentation to Optimise Active Lifestyles" by Zwolinsky S et al. Journal of Physical Activity \& Health

(C) 2016 Human Kinetics, Inc.

Public Health benefit. ${ }^{53}$ For instance, life expectancy across the world may rise by 0.68 years purely by eliminating physical inactivity. ${ }^{54}$

Effective policy and intervention may seek control exposure to inactivity and sedentary behaviour. Fundamentally, there are two ways to achieve this; (i) safeguarding vulnerable individuals through a 'high-risk- approach and (ii) controlling the causes of incidence through a population approach. ${ }^{55}$ The 'high-risk' approach will likely result in policy and interventions suitable for the individual, but won't address the underlying causes. While interventions are encouraged to emphasise the determinants of sedentary behaviour and develop tools to self-monitor/goal set, ${ }^{32}$ it is hard to convince people to 'step out of line' with their peers and this is what the individual strategy requires them to do. ${ }^{55}$ Therefore, policies need to do more to help individuals overcome internal and external barriers that currently compromise optimal lifestyles. ${ }^{56}$

In its traditional Public Health form, population strategies involve mass environmental control to alter societal norms, attempting to remove the underlying causes of the problem and improve the conditions for changing behaviour. ${ }^{55}$ This could be achieved by removing chairs from a desk or seated areas $^{3}$ rather than relying on individual decision-making. Nevertheless, one of the main drawbacks to this approach is that environmental approaches frequently only offer small benefits to the individual. For optimal effect, these approaches should be offered alongside, rather than instead of each other. The overarching priority should be to understand and control the causes of incidence.

The findings of this study should be interpreted within its limitations. The restricted demographic information and use of a non-probability sample may be subject to volunteer bias, which could threaten external validity. Similarly, subjectively measured physical activity and sitting time is subject to ascertainment bias through socially desirable responses. ${ }^{57}$ 
"Physical Activity and Sedentary Behaviour Clustering: Segmentation to Optimise Active Lifestyles" by Zwolinsky S et al. Journal of Physical Activity \& Health

(C) 2016 Human Kinetics, Inc.

Further, sIPAQ simply measures weekday sitting and recent evidence suggests that it likely underestimates this behaviour. ${ }^{11,47}$ Therefore, the use of a direct or objective measure that assesses the domains of physical activity and sitting time would have been preferable. ${ }^{47}$ However, given the size of the study, objective measurement was not practical. Further, cluster analysis is a data-driven dimension reduction technique; therefore generalizability beyond this sample should be treated with caution. However, these limitations should be considered against the study strengths, including the large sample size. Our cluster solution exhibited a high degree of within cluster homogeneity and between cluster heterogeneity. Also, results can be considered parsimonious and comprehensible given the number of clusters that were identified and the variables used to create them.

This research may be important for the Public Health system as it specifies clusters of individuals in need of distinctive strategies to help manage their unhealthy behaviours. Older adults, women and individuals seemingly less deprived than those in the most active clusters reported the most deleterious profiles. Nevertheless, regardless of activity status, modern lifestyles dictate that individuals are likely to spend a considerable portion of the day being sedentary. At present, Public Health policy advocates that adults should achieve $150+$ minutes of MVPA each week and limit time spent being sedentary, ${ }^{6}$ yet over half the UK adult population continue failing to meet these standards. Achieving the MVPA guidelines are likely to be aspirational - at best - for those who are currently 'Sedentary \& Low Active'. Therefore, physical activity advocates should exercise caution when promoting this universal message. Policy or intervention that helps replace sedentary time with light intensity activity in 'Sedentary \& Low Active' groups may be more rational and achievable, and can still generate substantial health benefit. ${ }^{35-37}$ 
"Physical Activity and Sedentary Behaviour Clustering: Segmentation to Optimise Active Lifestyles" by Zwolinsky S et al. Journal of Physical Activity \& Health

(c) 2016 Human Kinetics, Inc.

\section{Acknowledgments}

The authors would like to thank the participants who engaged the study and colleagues from

Leeds City Council for their contributions to the research including Rachel Brighton, Sue Haig and Mark Allman. We would also like to thank Sport England for funding this project through the Lottery supported Get Healthy, Get Active portfolio.

\section{Funding}

This study was part of the 'Leeds Let's Get Active' study funded by Sport England and Leeds Health and Wellbeing Board. 
"Physical Activity and Sedentary Behaviour Clustering: Segmentation to Optimise Active Lifestyles" by Zwolinsky S et al. Journal of Physical Activity \& Health

(C) 2016 Human Kinetics, Inc.

\section{REFERENCES}

1. Franklin B. Health implications of low cardiorespiratory fitness, too little exercise, and too much sitting time: changing paradigms and perceptions. Am J Health Promot. 2011;24(4):exiexv.

2. Owen N, Bauman A, Brown W. Too much sitting: a novel and important predictor of chronic disease risk? Br J Sports Med. 2009;43(2):81-3.

3. Owen N, Sugiyama T, Eakin EE, Gardiner PA, Tremblay MS, Sallis JF. Adults' sedentary behavior determinants and interventions. Am J Prev Med. 2011;41(2):189-96.

4. Katzmarzyk PT, Church TS, Craig CL, Bouchard C. Sitting time and mortality from all causes, cardiovascular disease, and cancer. Med Sci Sports Exerc. 2009;41(5):998-1005.

5. Tremblay MS, Colley RC, Saunders TJ, Healy GN, Owen N. Physiological and health implications of a sedentary lifestyle. Appl Physiol Nutr Metab. 2010;35(6):725-40.

6. Department of Health. Start Active, Stay Active: A report on physical activity for health from the four home countries" Chief Medical Officers. London: Department of Health; 2011.

7. Matthews CE, Chen KY, Freedson PS, et al. Amount of time spent in sedentary behaviors in the United States, 2003-2004. Am J Epidemiol. 2008;167(7):875-81.

8. Joint Health Surveys Unit. Health Survey for England 2008: Physical Activity and Fitness. The Information Centre: Leeds; 2010.

9. Ford ES, Caspersen CJ. Sedentary behaviour and cardiovascular disease: a review of prospective studies. Int J Epidemiol. 2012;41(5):1338-53.

10. Mansoubi M, Pearson N, Biddle SJ, Clemes S. The relationship between sedentary behaviour and physical activity in adults: a systematic review. Prev Med. 2014;69:28-35.

11. Bauman A, Ainsworth BE, Sallis JF, et al. The descriptive epidemiology of sitting. A 20-country comparison using the International Physical Activity Questionnaire (IPAQ). Am J Prev Med. 2011;41(2):228-35.

12. Bouchard C, Blair SN, Katzmarzyk PT. Less Sitting, More Physical Activity, or Higher Fitness? Mayo Clin Proc. 2015.

13. Sedentary Behaviour Research Network. Letter to the Editor: standardized use of the terms "sedentary" and "sedentary behaviours". Applied Physiology, Nutrition and Metabolism. 2012;37:540-2.

14. Chastin SF, Granat MH. Methods for objective measure, quantification and analysis of sedentary behaviour and inactivity. Gait Posture. 2010;31(1):82-6.

15. Hamilton MT, Hamilton DG, Zderic TW. Role of low energy expenditure and sitting in obesity, metabolic syndrome, type 2 diabetes, and cardiovascular disease. Diabetes. 2007;56(11):2655-67.

16. Thorp AA, Kingwell BA, English $\mathrm{C}$, et al. Alternating Sitting and Standing Increases the Workplace Energy Expenditure of Overweight Adults. J Phys Act Health. 2015. 
"Physical Activity and Sedentary Behaviour Clustering: Segmentation to Optimise Active Lifestyles" by Zwolinsky S et al. Journal of Physical Activity \& Health

(C) 2016 Human Kinetics, Inc.

17. Edwardson CL, Gorely T, Davies MJ, et al. Association of sedentary behaviour with metabolic syndrome: a meta-analysis. PLoS One. 2012;7(4):e34916.

18. Proper KI, Singh AS, van Mechelen W, Chinapaw MJ. Sedentary behaviors and health outcomes among adults: a systematic review of prospective studies. Am J Prev Med. 2011;40(2):174-82.

19. Wilmot EG, Edwardson CL, Achana FA, et al. Sedentary time in adults and the association with diabetes, cardiovascular disease and death: systematic review and meta-analysis. Diabetologia. 2012;55(11):2895-905.

20. van Uffelen JG, Wong J, Chau JY, et al. Occupational sitting and health risks: a systematic review. Am J Prev Med. 2010;39(4):379-88.

21. Healy GN, Matthews CE, Dunstan DW, Winkler EA, Owen N. Sedentary time and cardiometabolic biomarkers in US adults: NHANES 2003-06. Eur Heart J. 2011;32(5):590-7.

22. Healy GN, Dunstan DW, Salmon J, Shaw JE, Zimmet PZ, Owen N. Television time and continuous metabolic risk in physically active adults. Med Sci Sports Exerc. 2008;40(4):639-45.

23. Zwolinsky S, Pringle A, Daly-Smith A, McKenna J, Robertson S, White A. Associations between daily sitting time and the combinations of lifestyle risk factors in men. Journal of Men's Health. 2012;9(4):261-7.

24. Chau JY, Grunseit AC, Chey T, et al. Daily sitting time and all-cause mortality: a meta-analysis. PLoS One. 2013;8(11):e80000.

25. Dunstan DW, Barr EL, Healy GN, et al. Television viewing time and mortality: the Australian Diabetes, Obesity and Lifestyle Study (AusDiab). Circulation. 2010;121(3):384-91.

26. Patel AV, Bernstein L, Deka A, et al. Leisure time spent sitting in relation to total mortality in a prospective cohort of US adults. Am J Epidemiol. 2010;172(4):419-29.

27. Kim H, Iwasaki K, Miyake T, Shiozawa T, Nozaki S, Yajima K. Changes in bone turnover markers during 14-day 6 degrees head-down bed rest. J Bone Miner Metab. 2003;21(5):311-5.

28. Smith SM, Davis-Street JE, Fesperman JV, et al. Evaluation of treadmill exercise in a lower body negative pressure chamber as a countermeasure for weightlessness-induced bone loss: a bed rest study with identical twins. J Bone Miner Res. 2003;18(12):2223-30.

29. Hu FB, Li TY, Colditz GA, Willett WC, Manson JE. Television watching and other sedentary behaviors in relation to risk of obesity and type 2 diabetes mellitus in women. Jama. 2003;289(14):1785-91.

30. Biddle SJ. Fit or sit? Is there a psychology of sedentary behaviour? Sport \& Exercise Psychology Review. 2011;7(2):5-10.

31. Puig-Ribera A, Martinez-Lemos I, Gine-Garriga M, et al. Self-reported sitting time and physical activity: interactive associations with mental well-being and productivity in office employees. BMC Public Health. 2015;15:72.

32. Martin A, Fitzsimons C, Jepson R, et al. Interventions with potential to reduce sedentary time in adults: systematic review and meta-analysis. Br J Sports Med. 2015. 
"Physical Activity and Sedentary Behaviour Clustering: Segmentation to Optimise Active Lifestyles" by Zwolinsky S et al. Journal of Physical Activity \& Health

(C) 2016 Human Kinetics, Inc.

33. Biddle SJ, Marshall SJ, Gorely T, Cameron N. Temporal and environmental patterns of sedentary and active behaviors during adolescents' leisure time. Int J Behav Med. 2009;16(3):278-86.

34. Mutz D, Roberts C, Vuuren C. Reconsidering the displacement hypothesis: televisions influence on childrens time use. Communication Research. 1993;20:51-75.

35. Healy GN, Dunstan DW, Salmon J, et al. Objectively measured light-intensity physical activity is independently associated with 2-h plasma glucose. Diabetes Care. 2007;30(6):1384-9.

36. Dunstan DW, Kingwell BA, Larsen R, et al. Breaking up prolonged sitting reduces postprandial glucose and insulin responses. Diabetes Care. 2012;35(5):976-83.

37. Carson V, Ridgers ND, Howard BJ, et al. Light-intensity physical activity and cardiometabolic biomarkers in US adolescents. PLoS One. 2013;8(8):e71417.

38. Pearson N, Braithwaite RE, Biddle SJ, van Sluijs EM, Atkin AJ. Associations between sedentary behaviour and physical activity in children and adolescents: a meta-analysis. Obes Rev. 2014;15(8):666-75.

39. National Institute for Health and Care Excellence. Behaviour change: individual approaches. London: National Institute for Health and Care Excellence,; 2014.

40. Bowles HR. Measurement of active and sedentary behaviors: closing the gaps in self-report methods. J Phys Act Health. 2012;9 Suppl 1:S1-4.

41. Zwolinsky S, McKenna J, Pringle A, Widdop P, Griffiths C. Physical activity assessment for public health: efficacious use of the single-item measure. Public Health. 2015;129(12):1630-6.

42. McLennan D, Barnes H, Noble M, Davies J, Garratt E. The English Indices of Deprivation 2010. In: Department for Communities and Local Government, ed. London2011.

43. The English indices of deprivation 2010. Statistical release, 2011. at https://www.gov.uk/government/uploads/system/uploads/attachment data/file/6871/187 1208.pdf.)

44. Craig CL, Marshall AL, Sjostrom M, et al. International physical activity questionnaire: 12country reliability and validity. Med Sci Sports Exerc. 2003;35(8):1381-95.

45. Grimm EK, Swartz AM, Hart T, Miller NE, Strath SJ. Comparison of the IPAQ-Short Form and accelerometry predictions of physical activity in older adults. J Aging Phys Act. 2012;20(1):6479.

46. IPAQ Research Committee. Guidelines for Data Processing and Analysis of the International Physical Activity Questionnaire (IPAQ) - Short and Long Forms. In: International Physical Activity Questionnaire, ed.2005.

47. Chastin SF, Culhane B, Dall PM. Comparison of self-reported measure of sitting time (IPAQ) with objective measurement (activPAL). Physiol Meas. 2014;35(11):2319-28.

48. Hofstetter H, Dusseldorp E, van Empelen P, Paulussen TW. A primer on the use of cluster analysis or factor analysis to assess co-occurrence of risk behaviors. Prev Med. 2014;67:1416.

49. Schwarz G. Estimating the Dimension of a Model. The Annals of Statistics. 1978;6(2):461-4. 
"Physical Activity and Sedentary Behaviour Clustering: Segmentation to Optimise Active Lifestyles" by Zwolinsky S et al. Journal of Physical Activity \& Health

(C) 2016 Human Kinetics, Inc.

50. Yates $T$, Khunti K, Wilmot EG, et al. Self-reported sitting time and markers of inflammation, insulin resistance, and adiposity. Am J Prev Med. 2012;42(1):1-7.

51. Stephens BR, Granados K, Zderic TW, Hamilton MT, Braun B. Effects of 1 day of inactivity on insulin action in healthy men and women: interaction with energy intake. Metabolism. 2011;60(7):941-9.

52. Ford E, Greenlund K, Hong Y. Ideal cardiovascular health and mortality from all causes and diseases of the circulatory system among adults in the United States. Circulation. 2012;125(8):987-95.

53. Ekelund $\mathrm{U}$, Ward HA, Norat $\mathrm{T}$, et al. Physical activity and all-cause mortality across levels of overall and abdominal adiposity in European men and women: the European Prospective Investigation into Cancer and Nutrition Study (EPIC). Am J Clin Nutr. 2015;101(3):613-21.

54. Lee IM, Shiroma EJ, Lobelo F, Puska P, Blair SN, Katzmarzyk PT. Effect of physical inactivity on major non-communicable diseases worldwide: an analysis of burden of disease and life expectancy. Lancet. 2012;380(9838):219-29.

55. Rose G. Sick individuals and sick populations. Int J Epidemiol. 2001;30(3):427-32; discussion 33-4.

56. Pronk NP, Peek CJ, Goldstein MG. Addressing multiple behavioral risk factors in primary care. A synthesis of current knowledge and stakeholder dialogue sessions. Am J Prev Med. 2004;27(2 Suppl):4-17.

57. Adams SA, Matthews CE, Ebbeling CB, et al. The effect of social desirability and social approval on self-reports of physical activity. Am J Epidemiol. 2005;161(4):389-98. 
"Physical Activity and Sedentary Behaviour Clustering: Segmentation to Optimise Active Lifestyles" by Zwolinsky S et al. Journal of Physical Activity \& Health

(C) 2016 Human Kinetics, Inc.

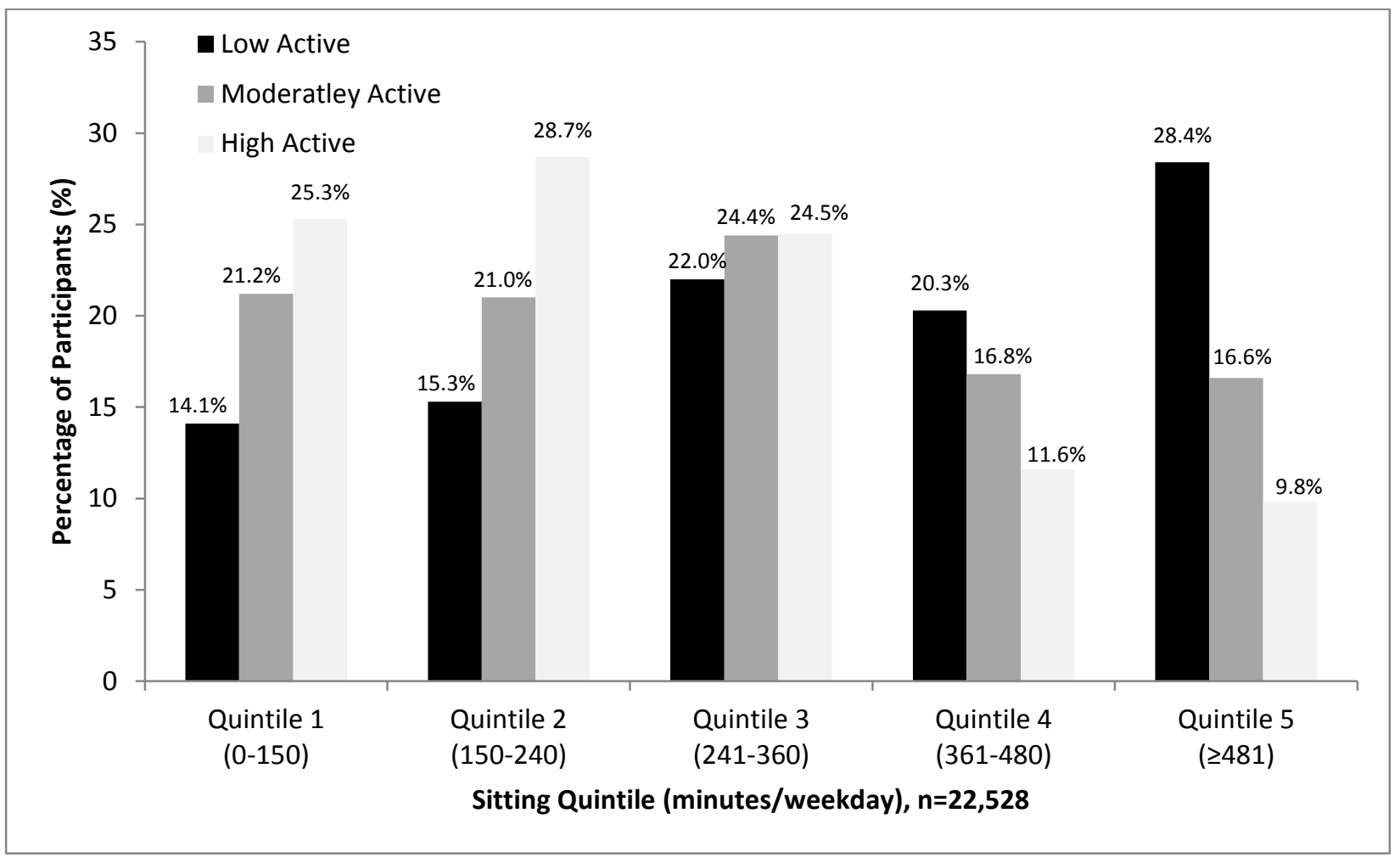

Figure 1: Sitting time and activity category combinations 
"Physical Activity and Sedentary Behaviour Clustering: Segmentation to Optimise Active Lifestyles" by Zwolinsky S et al. Journal of Physical Activity \& Health

(C) 2016 Human Kinetics, Inc.

Table 1: Socio demographic characteristics of participants

\% Participants (n)

\section{Socio Demographics}

Gender $(n=22,813)$

Male

$36.8(8,408)$

Female

$63.1(14,405)$

Age $(n=22,836)$

$16-25$

$20.6(4,700)$

26-35

$27.4(6,250)$

36-45

$22.7(5,178)$

46-55

$13.3(3,037)$

56-56

$9.5(2,179)$

$66-75$

$5.5(1,253)$

$76+$

$1.0(239)$

IMD Deciles $(n=22,463)$

$1-<6.85$ (Least Deprived)

$10.1(2,258)$

$2-6.85$ to 9.72

$10.1(2,273)$

$3-9.73$ to 12.62

$10.3(2,320)$

$4-12.63$ to 16.44

$10.6(2,386)$

$5-16.45$ to 20.64

$9.9(2,227)$

$6-20.65$ to 26.97

$10.1(2,265)$

$7-26.98$ to 34.56

$9.9(2,218)$

$8-34.57$ to 45.88

$9.8(2,203)$

$9-45.89$ to 52.52

$9.7(2,182)$

10 - >52.52 (Most Deprived)

$9.5(2,131)$

Note: IMD = Indices of Multiple Deprivation, 
"Physical Activity and Sedentary Behaviour Clustering: Segmentation to Optimise Active Lifestyles" by Zwolinsky S et al. Journal of Physical Activity \& Health

(c) 2016 Human Kinetics, Inc.

Table 2: Cluster characteristics

\begin{tabular}{|c|c|c|c|}
\hline & Cluster 1 & Cluster 2 & Cluster 3 \\
\hline & Ambulatory \& Active & Moderation & Sedentary \& Low \\
\hline & $(n=6,254,27.7 \%)$ & $(n=10,028,44.4 \%)$ & Active \\
\hline & & & $(n=6,286,27.9 \%)$ \\
\hline \multirow[t]{2}{*}{ Sitting Quintile } & Quintile 2 (151-240 & Quintile 3 (241-360 & Quintile 5 (481+ \\
\hline & Minutes/Weekday) & Minutes/Weekday) & Minutes/Weekday) \\
\hline Total Vigorous & 2,400 & 460 & 24 \\
\hline Activity & MET.min.wk ${ }^{-1}$ & MET.min.wk ${ }^{-1}$ & MET.min.wk-1 \\
\hline Total Moderate & 1,371 & 284 & 32 \\
\hline Activity & MET.min.wk ${ }^{-1}$ & MET.min. wk $^{-1}$ & MET.min.wk ${ }^{-1}$ \\
\hline Total Walking & 2,104 & 852 & 168 \\
\hline Activity & MET.min.wk-1 & MET.min. wk $^{-1}$ & MET.min.wk-1 \\
\hline Physical Activity & High & Moderate & Low \\
\hline Category & & & \\
\hline
\end{tabular}


"Physical Activity and Sedentary Behaviour Clustering: Segmentation to Optimise Active Lifestyles" by Zwolinsky S et al. Journal of Physical Activity \& Health

(C) 2016 Human Kinetics, Inc.

Table 3: Between Group Differences in Cluster Demographics

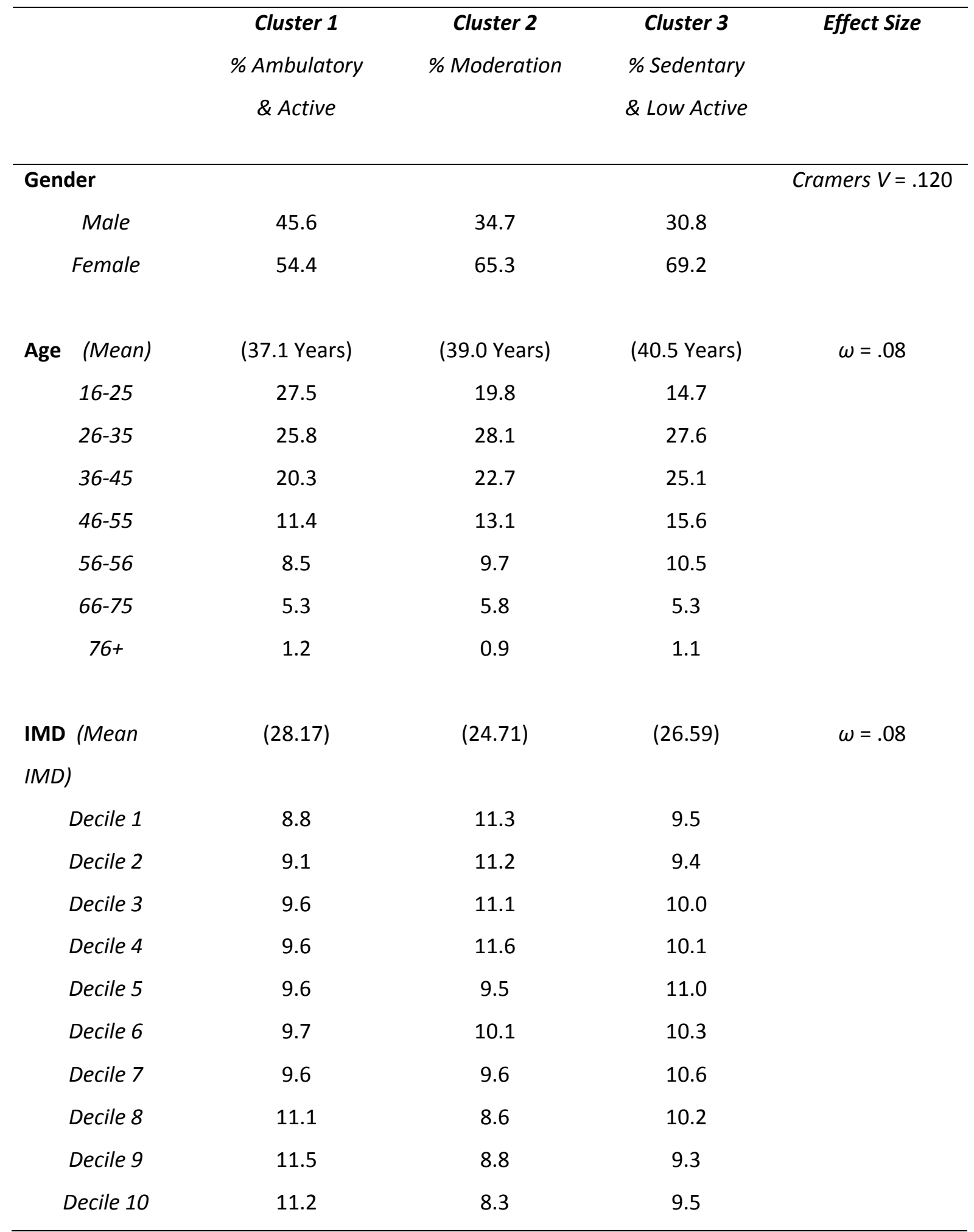

